\title{
Ultrasound-Guided Percutaneous AI Pulley Release by Acupotomy (Needle-Knife): A Cadaveric Study of Safety and Efficacy
}

\author{
Jiaxuan Yang ${ }^{\prime}, *$, Bin $\mathrm{Ma}^{2}{ }^{2}$, Hao Zhong', Yue Zhang', Jiang Zhu', Yong $\mathrm{Ni}^{\prime}$ \\ University, Suzhou, Jiangsu, People's Republic of China \\ *These authors contributed equally to this work \\ Correspondence: Jiang Zhu; Yong Ni, Tel +86 18013575733, Email zhujiangsz@I26.com; niyong2 I48@I63.com
}

'Pain Department, The Second Affiliated Hospital of Soochow University, Suzhou, Jiangsu, People's Republic of China; ${ }^{2}$ Medical College, Soochow

\begin{abstract}
Purpose: This study was to assess the safety and effectiveness of ultrasound-guided percutaneous A1 pulley release by acupotomy on unembalmed cadavers.

Materials and Methods: Sixty digits (from six cadavers, three male and three female) were split into two groups using stratified randomization. All procedures were completed by a single doctor with rich experience in ultrasound-guided treatment. In the acupotomy group, the A1 pulley was released under ultrasound-guided by a needle-knife; while in the needle group, the A1 pulley was released under ultrasound-guided by a 21-gauge needle. Two groups completed six thumbs and 24 fingers, respectively. Another anatomist, blinded to the two techniques, assessed the safety, including the minimum distance between the incision and the neurovascular; flexor tendon, neurovascular and A2 pulley injury or not. Completeness release of the A1 pulley was recorded as effectiveness.

Results: No neurovascular or A2 pulley injuries were recorded. However, the incision of the thumb in both groups biased to the radial side $(P<0.05)$, while the incision of the finger biased to the ulnar side $(P<0.05)$. No significant flexor tendon injury was found, and only five cases $(16.7 \%)$ had minor scratches in the acupotomy group; while in the needle group, 15 cases had minor scratches and lacerations occurred in three cases. The flexor tendon injury rate was $60 \%$. Compared with the needle, ultrasound-guided acupotomy release is safer $(P<0.05)$. The ultrasound-guided acupotomy technique was significantly more likely to result in a complete A1 pulley release compared to the needle technique (28 of $30[93.3 \%]$ versus 11 of $30[36.7 \%] ; P<0.05$ ).

Conclusion: Ultrasound-guided percutaneous A1 pulley release by acupotomy is a safe and effective technique. When releasing the thumb by ultrasound-guided, be careful not to bias to the radial side to avoid neurovascular injury, while when releasing a finger, be careful not to bias to the ulnar side.
\end{abstract}

Keywords: ultrasonography, acupotomy, trigger finger, anatomical study

\section{Introduction}

Trigger finger is a common cause of hand disability and pain. The acquired trigger finger has a lifetime prevalence of $2 \%$, and incidence is estimated at 28 per 100,000 in the adult population. ${ }^{1}$ Although the pathogenesis is incompletely clear and multifactorial, the most common cause of trigger finger is that the thickened flexor tendon stuck to the thickened first annular (A1) pulley. $^{2}$ Symptomatic trigger finger refers to non-surgical treatment, physical therapy, and corticosteroid injection, with an effective rate of $45-80 \%{ }^{3}$ Open surgical release is indicated for refractory symptoms, the effective rate of A1 pulley release is between $90 \%$ and $100 \% .{ }^{4}$ However, surgical complications, including infection, weakness, nerve injury, stiffness, flexor tendon bow stringing, and scarring, have been reported in an incidence of up to $12 \% .{ }^{5}$ In 1958 , Lorthioir ${ }^{6}$ introduced a technique for the percutaneous release of the A1 pulley using a special cutting device. Since then, many studies have used different cutting devices for percutaneous release of the A1 pulley, such as a 21-Gauge Needle, ${ }^{7}$ Stab Knife, ${ }^{8}$ and so on. Recently, it has also been reported that the use of acupotomy percutaneous release of the A1 pulley in the treatment of trigger finger had achieved good results. ${ }^{9}$ 
However, continued safety concerns, including the risk of flexor tendon and neurovascular injury, may prevent more widespread adoption of this technique. ${ }^{10}$ Real-time ultrasound-guided can potentially address these safety concerns through direct visualization of "High Risk" organizations. ${ }^{11}$ At present, there are few reports on the cadaveric study of ultrasound-guided percutaneous release of the A1 pulley by acupotomy. In this study, 60 digits of unembalmed cadaveric specimens were divided into two groups, after simulating local anesthesia, they were completed by a single doctor with rich experience in ultrasound-guided treatment, and then another anatomist, who was blinded to the method, evaluated the safety and effectiveness of the two techniques.

\section{Materials and Methods}

\section{General}

This study has been performed in accordance with the principles stated in the Declaration of Helsinki. This is not a human study and anatomical studies using cadavers donot require the formal review of the institutional review board (IRB) and, thus, our IRB approved the exemption from formal review for this study. All of the cadavers used in this study were legally donated to the Medical College of Soochow University. Six fresh frozen adult cadavers were independently chosen by the anatomist at Medical College of Soochow University. A total of 60 digits (including 12 thumbs and 48 fingers) from six unembalmed cadaver specimens (three male and three female) were assigned to the acupotomy group or needle group by stratified randomization, which ensured that each technique completed half of the fingers and thumbs from three males and three females. All digits were normal, without trauma, deformity, or having any previous operation.

\section{Equipment}

All procedures were performed using a VINNO 6 ultrasound machine fitted with the linear probe X6-16L (frequency range is $6.5-18 \mathrm{Mhz}$ ) with a $1.5 \mathrm{~cm}$ footprint (VINNO Technology, Suzhou, China). A Hanzhang needle-knife $(0.8 \mathrm{~mm}$ diameter and $40 \mathrm{~mm}$ length, Suzhou East Acupuncture Instruments Factory) and 21-Gauge needle (0.8 $\mathrm{mm}$ in diameter, $38 \mathrm{~mm}$ in length) were used ${ }^{9,12}$ in this study (Figure 1A).

\section{Relevant Sonographic Anatomy}

Under high-frequency ultrasound scanning, a homogeneous hypoechoic can be visualized at the metacarpophalangeal joint, which is the A1 pulley, and the flexor tendon appears as a slightly hyperechoic fibrillar (Figure 2A). The average thickness of the normal A1 pulley on the long and short axis views is $0.5 \mathrm{~mm}$ (range $=0.4-0.6 \mathrm{~mm}$ ) and the length is about $10 \mathrm{~mm}(9.8-10.2 \mathrm{~mm}) .{ }^{11}$ The second annular (A2) pulley is in the middle of the proximal phalanx, which can be recognized as thin $(0.3-0.5 \mathrm{~mm})$ hypoechoic by ultrasound in the long-axis view. The neurovasculars are identified from both sides of the flexor tendon by ultrasound on the short-axis view ${ }^{13}$ (Figure 2B).

\section{Ultrasound-Guided Percutaneous AI Pulley Release Technique}

All 60 digits (including 12 thumbs and 48 fingers) were completed by a single doctor with more than 3 years of experience in ultrasound-guided treatment. 1) The acupotomy group: The hand was placed palm-up, and the target finger was straightened and coated with coupling agent. The high-frequency probe coated with the coupling agent was wrapped in a sterile plastic film. The probe was placed on the long axis in the middle of the flexor tendon and the distal part of the metacarpophalangeal joint. The insertion point was about $0.5 \mathrm{~cm}$ from the proximal edge of the probe (Figure 1B). 21-Gauge syringe aspirates 1\% lidocaine $2 \mathrm{~mL}$, after simulating local anesthesia, under ultrasound-guided the needle was inserted close to the A1 pulley, and then about $1 \mathrm{~mL}$ of water was injected to separate the A1 pulley and the surrounding tissue. A needle-knife with a diameter of $0.8 \mathrm{~mm}$ was used to pierce the skin through the same puncture site, and then advanced into the A1 pulley horizontally under ultrasound-guided (Figure 3A). The long and short axis views of the ultrasound confirmed that the needle-knife maintained the midline and avoided the digital neurovascular (Figure 3B). And then, under the guidance of the long axis of ultrasound in realtime, a longitudinal horizontal cut the A1 pulley from proximal to distal. The procedure was considered complete when the A1 pulley was discontinuous under ultrasonic visualization, or the gravel feeling of the A1 pulley could no longer be appreciated. ${ }^{9,12}$ 2) The needle group: A 21-Gauge needle replaced the needle-knife, and the remaining steps were the same as in the acupotomy group (Figure 1C). 

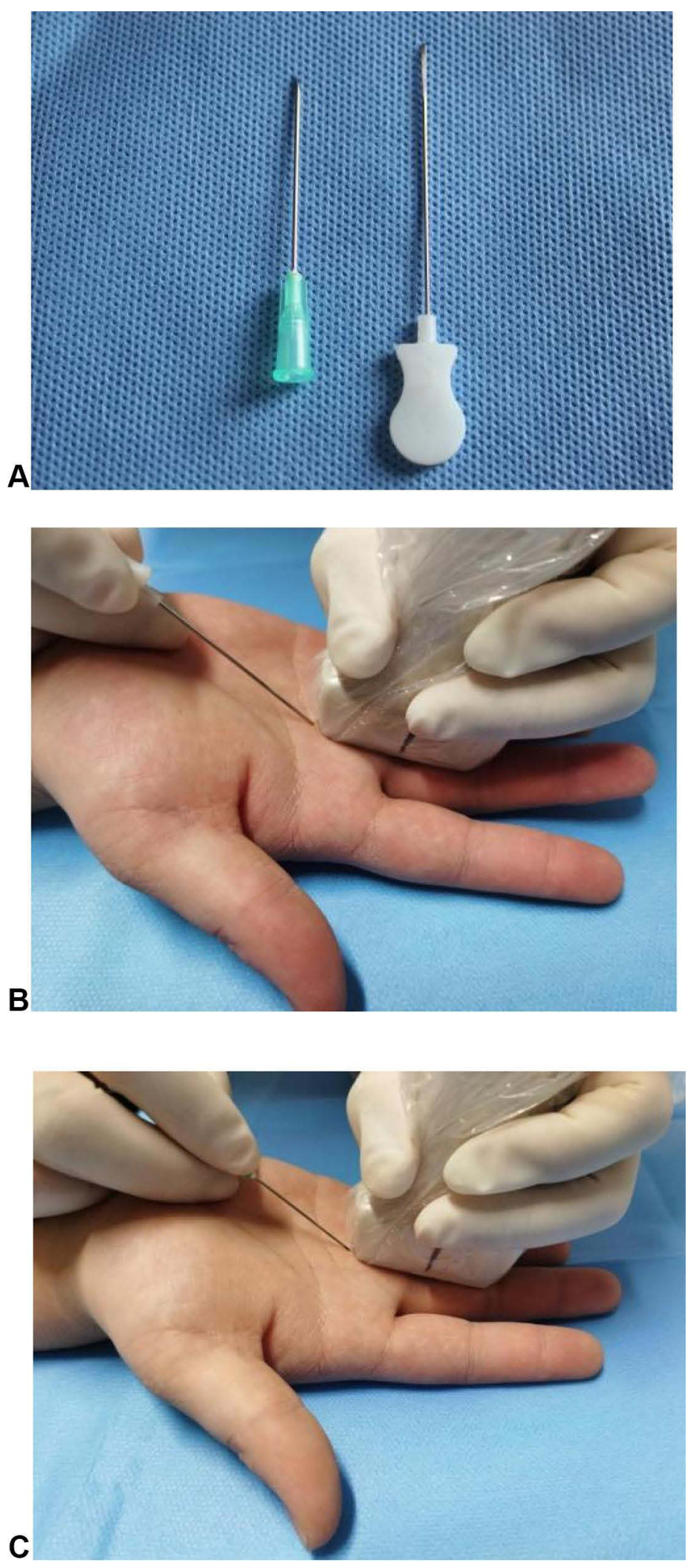

Figure I (A) On the left is a $2 \mathrm{I}-$-Gauge needle $(0.8 \mathrm{~mm}$ in diameter, $38 \mathrm{~mm}$ in length) and on the right is a Hanzhang needle-knife (0.8 mm diameter and $40 \mathrm{~mm}$ length); (B) placement of probe and needle-knife under ultrasound-guided percutaneous AI pulley release by acupotomy; (C) placement of probe and needle under ultrasound-guided percutaneous AI pulley release by 21 -Gauge needle. 

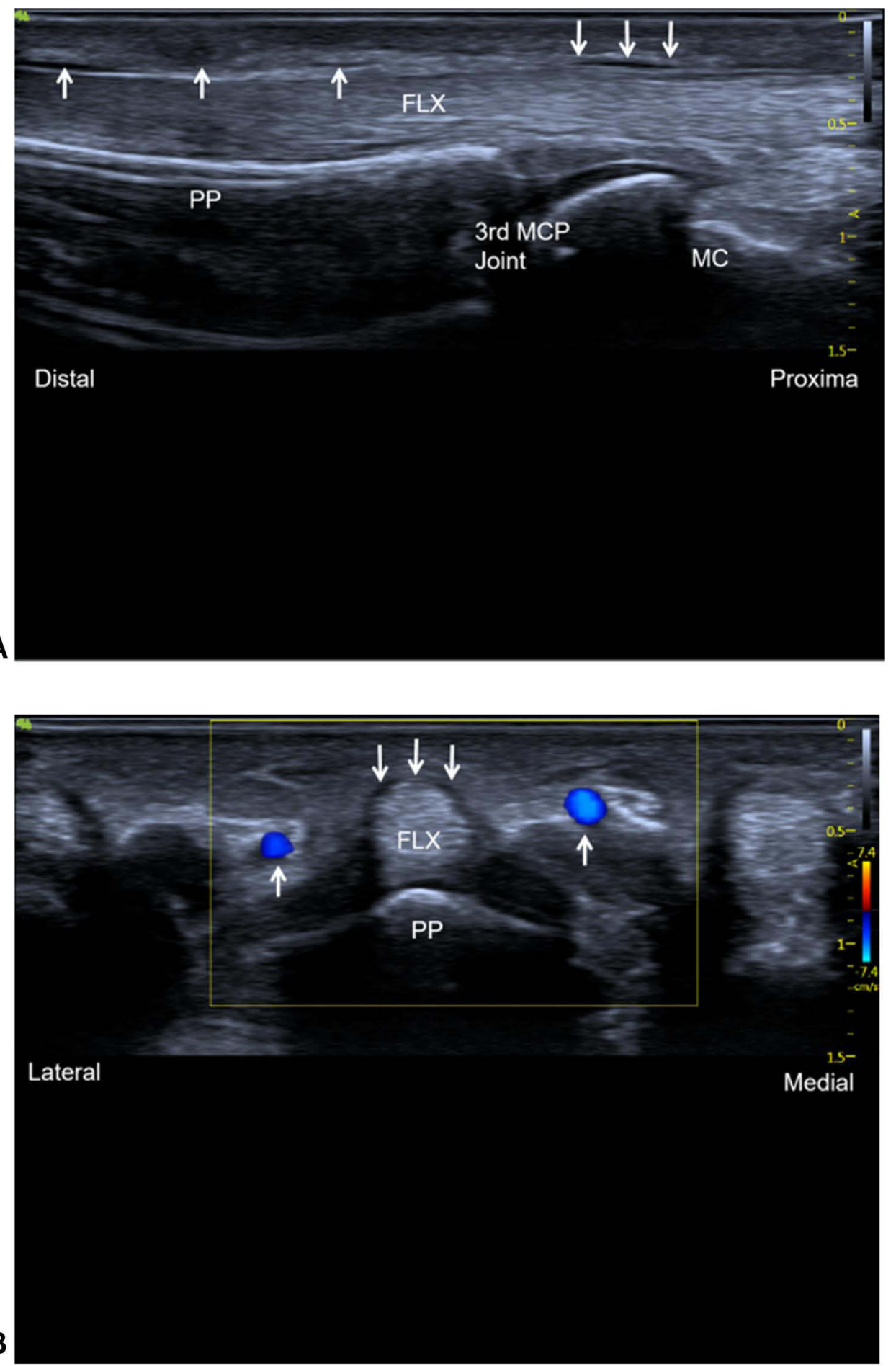

Figure 2 (A) Long-axis view of the FLX showing the Al pulley at the level of the third MCP joint. The thin hypoechoic Al pulley is identified by $\downarrow$, hypoechoicis $\mathrm{A} 2$ pulley is identified by $\uparrow$; (B) short-axis view of the FLX, the thin hypoechoic Al pulley is identified by $\downarrow$, the neurovasculars are identified by $\uparrow$.

Abbreviations: FLX, finger flexor tendons; 3rd MCP joint, the 3rd metacarpophalangeal joint; MC, metacarpal head; PP, proximal phalanx.

\section{Assessment}

All the digits were dissected and assessed by another anatomist with more than 3 years of experience, who was blinded to each technique. Each digit recorded the following data: the minimum distance between the incision and the neurovascular on both sides, and whether there was any neurovascular injury, tendon injury (no; minor scratches: surface scratches $<10 \%$ thickness of tendon; lacerations: lacerations $>10 \%$ thickness of tendon), ${ }^{14,15}$ and whether the A2 pulley was 

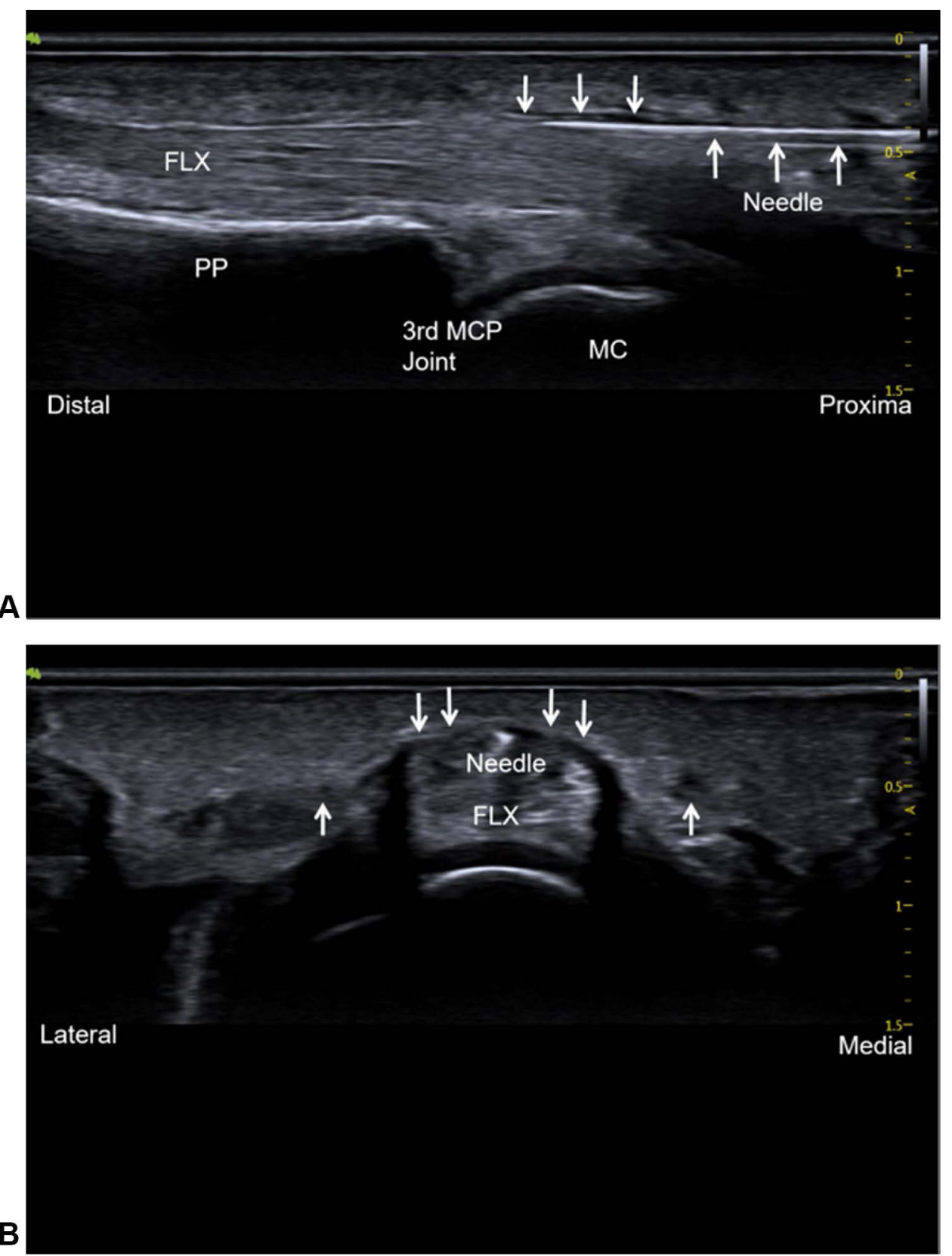

Figure 3 (A) Ultrasound-guided percutaneous AI pulley release by acupotomy. Long-axis view of the FLX show the AI pulley at the MCP joint, and the needle-knife advanced into the Al pulley horizontally under ultrasound-guided. The thin hypoechoic Al pulley is identified by $\downarrow$, the hyperechoic needle-knife identified by $\uparrow$; (B) shortaxis view of the FLX show the AI pulley, the thin hypoechoic Al pulley is identified by $\downarrow$, the hypoechoic neurovascular identified by $\uparrow$, the hyperechoic needle-knife is in the midline and avoids the neurovascular.

Abbreviations: FLX, finger flexor tendons; 3rd MCP joint, the 3rd metacarpophalangeal joint; MC, metacarpal head; PP, proximal phalanx.

injured (yes or no). The completeness release of the A1 pulley was regarded as the determination of treatment effectiveness (yes or no).

\section{Statistics}

The descriptive data were tested for normal distribution. Data were expressed as mean \pm standard deviation. The Student's $t$-test was used for the comparison between the two groups, and the Fisher exact test was used for the counting data. A $P$-value $\leq 0.05$ was considered as the level of significance. 
Table I The Minimum Distance Between the Incision and the Neurovascular of Thumbs

\begin{tabular}{|l|l|l|}
\hline Groups & The Radial Distance $\mathbf{( m m})$ & The Ulnar Distance $(\mathbf{m m})$ \\
\hline Needle group $(n=6)$ & $2.4 \pm 0.4$ & $4.9 \pm 0.4$ \\
Acupotomy group $(n=6)$ & $2.8 \pm 0.5$ & $4.4 \pm 0.4$ \\
\hline
\end{tabular}

Note: Data are presented as mean \pm SD.

Table 2 The Minimum Distance Between the Incision and the Neurovascular of Fingers

\begin{tabular}{|l|l|l|}
\hline Groups & The Radial Distance $\mathbf{( m m})$ & The Ulnar Distance $(\mathbf{m m})$ \\
\hline Needle group $(n=24)$ & $3.9 \pm 0.6$ & $3.4 \pm 0.7$ \\
Acupotomy group $(\mathrm{n}=24)$ & $3.9 \pm 0.6$ & $3.5 \pm 0.7$ \\
\hline
\end{tabular}

Note: Data are presented as mean \pm SD.

\section{Results}

\section{Release Safety}

Safety data statistics showed that 24 fingers and six thumbs were completed in the two groups, respectively, and there was no neurovascular or A2 pulley injury. The incision was mostly in the midline of the flexor tendon, which was still a certain distance from the neurovascular. Tables 1 and 2 show the measured distance between the incision and the radial and ulnar neurovascular of the thumbs and fingers, respectively. As shown in Table 1, acupotomy group $2.8 \pm 0.5 \mathrm{~mm}$ versus $4.4 \pm 0.4 \mathrm{~mm}$; needle group $2.4 \pm 0.4 \mathrm{~mm}$ versus $4.9 \pm 0.4 \mathrm{~mm}$, the incision of the thumb in both groups biased to the radial side $(P<0.05)$; while in Table 2, acupotomy group $3.9 \pm 0.6 \mathrm{~mm}$ versus $3.5 \pm 0.7 \mathrm{~mm}$; needle group $3.9 \pm 0.6 \mathrm{~mm}$ versus $3.4 \pm 0.7 \mathrm{~mm}$, the incision of the finger biased to the ulnar side $(P<0.05)$ (Figure 4$)$. As shown in Table 3, no significant flexor tendon injury was found, and only five cases (16.7\%) had minor scratches in the acupotomy group; while in the needle group, 15 cases had minor scratches and lacerations occurred in three cases, the flexor tendon injury rate is $60 \%$. Compared with the needle, the acupotomy guided by ultrasound was safer $(P<0.05)$.
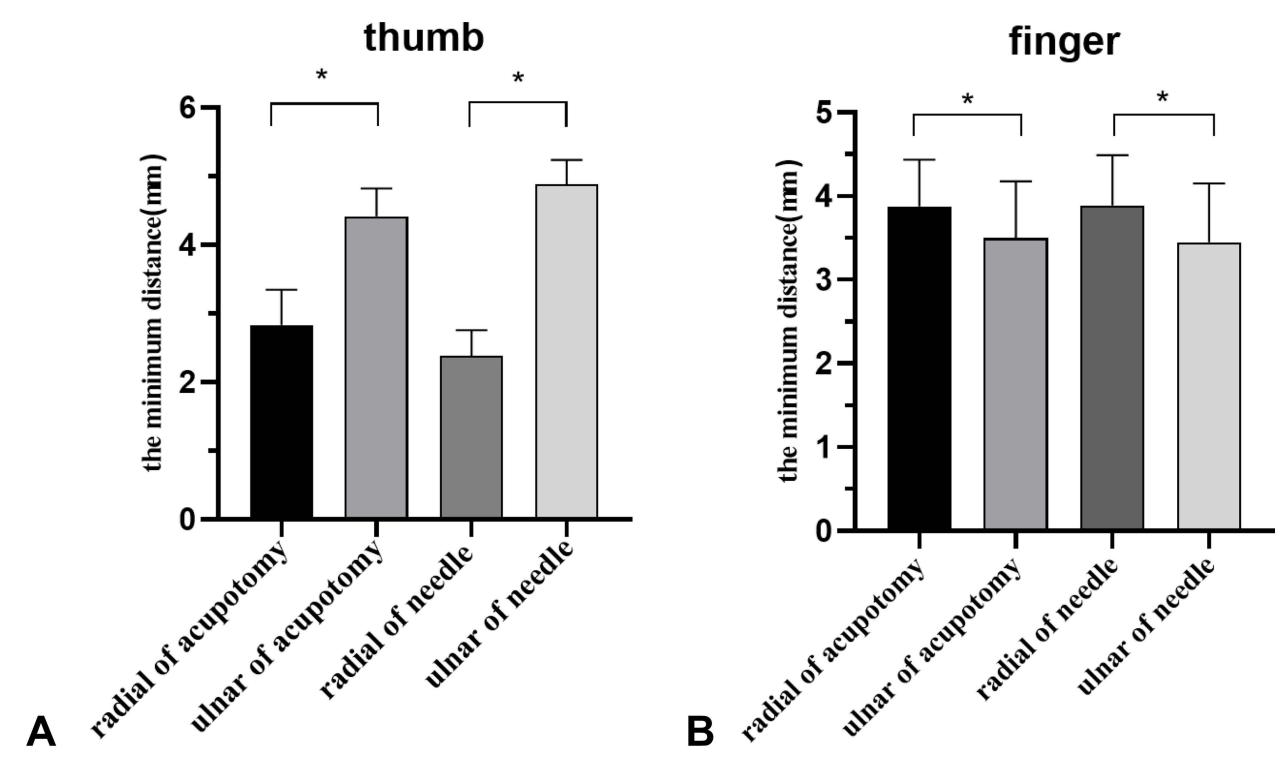

Figure 4 (A) The minimum distance between the incision and the radial and ulnar neurovascular of the thumbs; (B) The minimum distance between the incision and the radial and ulnar neurovascular of the fingers.

Note: * Statistically significant $(P<0.05)$ between-group differences. 
Table 3 Tendon Injury Rate in Two Groups $(n=30)$

\begin{tabular}{|c|c|c|c|c|}
\hline Groups & No, n (\%) & $\begin{array}{l}\text { Minor Scratches: }<10 \% \text { of Tendon } \\
\text { Thickness, } \mathbf{n}(\%)\end{array}$ & $\begin{array}{l}\text { Lacerations: > } 10 \% \text { of Tendon } \\
\text { Thickness, } \mathbf{n}(\%)\end{array}$ & $\begin{array}{l}\text { Tendon Injury } \\
\text { Rate, n (\%) }\end{array}$ \\
\hline Needle group & $12(40 \%)$ & 15 (50\%) & $3(10 \%)$ & $18(60 \%)$ \\
\hline Acupotomy group & 25 (83.3\%) & 5 (16.7\%) & 0 & 5 (16.7\%)* \\
\hline
\end{tabular}

Note: * Statistically significant difference between acupotomy group versus needle group $(P<0.05)$.

\section{Success of AI Pulley Release}

The effectiveness statistics showed that 28 cases of 30 digits in the needle knife group were completely released (93.3\%); Only 11 of the 30 digits in the needle group were completely released (36.7\%) (Figure 5). The ultrasound-guided acupotomy technique was significantly more likely to result in a complete pulley release compared to the needle technique (28 of 30 [93.3\%] versus 11 of 30 [36.7\%]; $P<0.05$ ) (Table 4$)$.

\section{Discussion}

This study compared the safety and effectiveness of ultrasound-guided acupotomy and needle in percutaneous release of the A1 pulley. Acupotomy is a combination of traditional Chinese acupuncture therapy and modern medical surgery. The main role of the needle knife is to release adhesion, improve blood circulation, and increase the metabolism of local paincausing substances. It is composed of three parts: tip, body and handle. It does not need to cut the skin, but only needs percutaneous release. ${ }^{9}$ In this study, a Hanzhang needle-knife with a $0.8 \mathrm{~mm}$ diameter and a $40 \mathrm{~mm}$ length was selected. After simulating local anesthesia, two different techniques were used to release the A1 pulley on unembalmed specimens. Ultrasound transmission requires a medium, and formalin or dry specimens can interfere with ultrasound scanning and guided release. Therefore, the unembalmed specimens were selected to simulate the real clinical situation as far as possible.

Both groups completed 24 fingers and six thumbs under real-time ultrasound-guided, respectively. After dissection, there was no neurovascular injury in both groups. The incision was mostly in the midline of the flexor tendon, and it was still some distance away from the neurovascular. Tables 1 and 2 show the measured distance between the incision and the radial and ulnar neurovascular for the thumbs and fingers, respectively. As shown in Table 1, acupotomy group $2.8 \pm 0.5 \mathrm{~mm}$ versus $4.4 \pm 0.4 \mathrm{~mm}$; needle group $2.4 \pm 0.4 \mathrm{~mm}$ versus $4.9 \pm 0.4 \mathrm{~mm}$, the distance between the thumb incision and the radial neurovascular was shorter in the two groups, with a statistically significant difference between the two groups $(P<0.05)$ (Figure 4$)$, the incision of the thumb in both groups biased to the radial side. Although not in the table, the distance from the incision to the radial neurovascular was less than or equal to $2 \mathrm{~mm}$ in three cases. While in Table 2, acupotomy group $3.9 \pm 0.6 \mathrm{~mm}$ versus $3.5 \pm 0.7 \mathrm{~mm}$; needle group $3.9 \pm 0.6 \mathrm{~mm}$ versus $3.4 \pm 0.7 \mathrm{~mm}$, the distance between the finger incision and the ulnar neurovascular was shorter in the two groups, with a statistically significant difference between two groups $(P<0.05)$ (Figure 4), the incision of the finger biased to the ulnar side. Although not in the table, the distance from the incision to the ulnar neurovascular was less than or equal to $2 \mathrm{~mm}$ in four cases. In some studies, the distance between the incision and the neurovascular less than or equal to $2 \mathrm{~mm}$ is defined as a significant risk of neurovascular injury. ${ }^{16}$ In addition, previous anatomical studies had shown that the neurovascular of the ring and middle fingers were located on either side of the finger and parallel to the longitudinal axis of the finger, while the long axis of the radial neurovascular of the index finger and the ulnar neurovascular of the little finger were obliquely located near the A1 pulley at the metacarpophalangeal joint. ${ }^{17}$ Besides the thumb is special and can not be completely flattened like other fingers, so the incision is more likely to be biased to the ulnar side. Therefore, we should pay attention to avoid the incision bias to the radial side when releasing the A1 pulley of thumb, and avoid the incision bias to the ulnar side when releasing the A1 pulley of the little finger. When releasing the A1 pulley under ultrasound-guided, under short axis scanning we can confirm whether the needle-knife is in the midline. At the same time, we can clearly see the neurovasculars on both sides. Excessive A2 pulley injury ( $>25 \%$ of the length) can cause flexor tendon bow stringing, which is a serious complication. ${ }^{18}$ No A2 pulley injury was observed in 

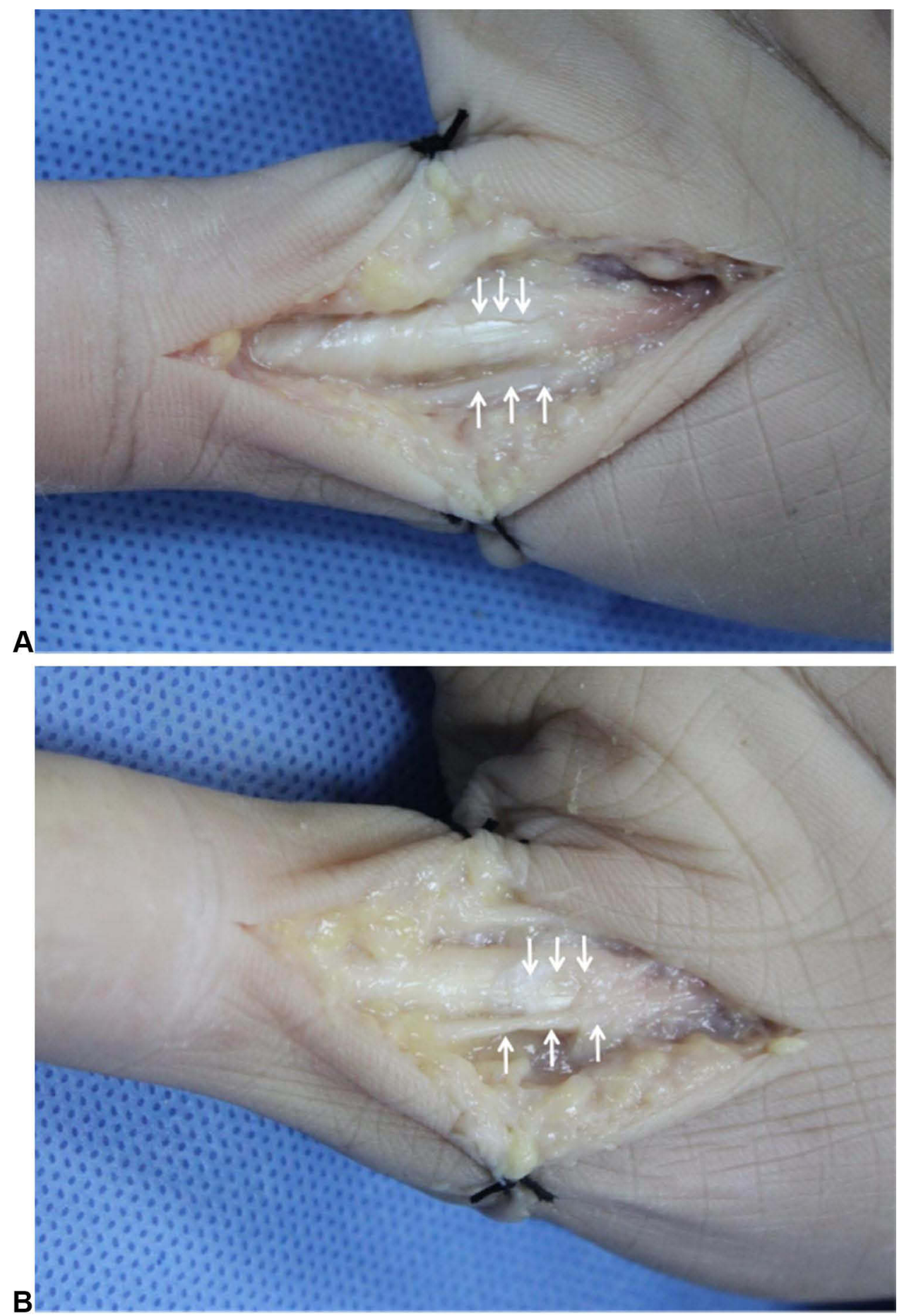

Figure 5 (A) In the acupotomy group the AI pulley was completely released and the incision was smooth; (B) in the needle group the AI pulley was only partially released with tendon injury; the AI pulley is identified by $\downarrow$, the neurovascular is identified by $\uparrow$.

either of the two groups. Previous studies had measured the length of the A1 pulley: thumb $5.30 \pm 0.53 \mathrm{~mm}$, index finger $6.32 \pm 0.17 \mathrm{~mm}$, middle finger $6.58 \pm 0.19 \mathrm{~mm}$, ring finger $6.32 \pm 0.20 \mathrm{~mm}$, little finger $5.30 \pm 0.49 \mathrm{~mm},{ }^{19}$ and the A1 pulley and A2 pulley can be clearly seen under ultrasound-guided. Therefore, it is safer to release under ultrasound-guided. Flexor tendon injury can lead to complications such as stiffness, pain, and adhesion. Therefore, flexor tendon injury should be avoided in the process of percutaneous release. ${ }^{16}$ As shown in Table 3, no significant flexor tendon injury was found, and only five cases (16.7\%) had minor scratches, which is less than $10 \%$ tendon 
Table 4 The Effective Rate in Two Groups $(n=30)$

\begin{tabular}{|l|l|l|}
\hline Groups & Completely(Effectiveness), n (\%) & Partly, n (\%) \\
\hline Needle group & $11(36.7 \%)$ & $19(63.3 \%)$ \\
Acupotomy group & $28(93.3 \%)^{*}$ & $2(6.7 \%)$ \\
\hline
\end{tabular}

Note: * Statistically significant difference between acupotomy group versus needle group $(P<0.05)$.

thickness in the acupotomy group, and the incision was smooth. In the needle group, 15 cases had minor scratches and tendon lacerations, which is more than $10 \%$ tendon thickness, occurred in three cases, the flexor tendon injury rate was $60 \%$ and the incision was serrated (Figure 5). Compared with the needle, the acupotomy guided by ultrasound was safer $(P<0.05)$.

An important anatomical basis for the effective treatment of trigger finger is the complete release of the A1 pulley. In the acupotomy group, 28 cases of 30 digits were completely released (93.3\%); Only 11 of the 30 digits in the needle group were completely released (36.7\%). Under ultrasound-guided we can directly visualize the A1 pulley, and the needle-knife was inserted horizontally at the proximal end of the A1 pulley. The A1 pulley was completely released by longitudinal cutting from the proximal end to the distal end (Figure 5). The ultrasound-guided acupotomy technique was significantly more likely to result in a complete pulley release compared to the needle technique (28 of 30 [93.3\%] versus 11 of 30 [36.7\%]; $P<0.05$ ) (Table 4). Our findings are consistent with previously published data on ultrasound-guided percutaneous release of the A1 pulley. One study evaluated the efficacy of ultrasound-guided percutaneous release of the A1 pulley with a 21-gauge needle for trigger fingers. All finger releases were considered "Partial". In clinical studies, $81.7 \%$ (49/60) of patients had the trigger finger completely removed immediately after surgery. ${ }^{12}$ Another prospective clinical study evaluated the efficacy of ultrasound-guided percutaneous A1 pulley release with the needle knife for trigger finger. The ultrasound-guided group (20 patients) had a significantly better grade postoperatively and reached $100 \%$ complete release in one time compared to the the traditional needle-knife group (21 patients) $(P<0.05)$. Moreover, no any complications occurred in the ultrasound-guided group. ${ }^{9}$

There are still some limitations in this study. Firstly, only 60 digits from six fresh frozen cadavers (three male and three female) were selected, and the sample size is small. Secondly, no thickening of the pulley and flexor tendon was found in ultrasound scanning, which is different from the ultrasound images of trigger finger. Clinically, ultrasound scanning can more clearly show the thickened A1 pulley, and the target is more clear. Thirdly, both groups were completed by the same doctor with more than 3 years of ultrasound-guided treatment experience. The ultrasound-guided technique needs a learning curve. ${ }^{18}$ There may be some differences in the results of operations by doctors with different experience. Fourthly, the measurement of the distance from the incision to the neurovascular after dissection, which may change the original distance. Therefore, there are some differences with the results of other previous studies, but it does not affect the safety results of this study.

\section{Conclusion}

In conclusion, our study shows that ultrasound-guided percutaneous A1 pulley release is a safe and effective technique. This study confirmed anatomically that ultrasound-guided acupotomy release has no neurovascular and tendon injury, which is safer than ultrasound-guided needle release. The ultrasound-guided acupotomy technique was significantly more likely to result in a complete A1 pulley release compared to the needle technique.

\section{Acknowledgments}

This study was partly supported by Medical Application Foundation of Suzhou (SYS2019074, SKY2021042, SYSD2019206, SYSD2020208); Young Medical Talents of Jiangsu Province (QNRC2016876); Health Talents Training Project of Gusu District (GSWS2019040); Research Foundation from the Second Affiliated Hospital of Soochow University (XKTJ-TD202008) and Jiangsu Key Laboratory of Neuropsychiatric Diseases (KJS2114). 


\section{Disclosure}

The authors report no conflicts of interest in this work.

\section{References}

1. David M, Rangaraju M, Raine A. Acquired triggering of the fingers and thumb in adults. BMJ. 2017;359:j5285. doi:10.1136/bmj.j5285

2. Fiorini HJ, Tamaoki MJ, Lenza M, et al. Surgery for trigger finger. Cochrane Database Syst Rev. 2018;2(2):CD009860. doi:10.1002/14651858. CD009860.pub2

3. Leow MQH, Hay ASR, Ng SL, et al. A randomized controlled trial comparing ketorolac and triamcinolone injections in adults with trigger digits. J Hand Surg Eur Vol. 2018;43(9):936-941. doi:10.1177/1753193418756808

4. Bruijnzeel H, Neuhaus V, Fostvedt S, et al. Adverse events of open A1 pulley release for idiopathic trigger finger. J Hand Surg Am. 2012;37 (8):1650-1656. doi:10.1016/j.jhsa.2012.05.014

5. Everding NG, Bishop GB, Belyea CM, et al. Risk factors for complications of open trigger finger release. Hand (N Y). 2015;10(2):297-300. doi:10.1007/s11552-014-9716-9

6. Lorthioir J. Surgical treatment of trigger-finger by a subcutaneous method. J Bone Joint Surg Am. 1958;40(4):793-795. doi:10.2106/00004623195840040-00003

7. Eastwood DM, Gupta KJ, Johnson DP. Percutaneous release of the trigger finger: an office procedure. J Hand Surg Am. 1992;17(1):114-117. doi:10.1016/0363-5023(92)90125-9

8. Abdoli A, Asadian M, Banadaky SHS, et al. A cadaveric assessment of percutaneous trigger finger release with $15^{\circ}$ stab knife: its effectiveness and complications. J Orthop Surg Res. 2021;16(1):426. doi:10.1186/s13018-021-02566-4

9. Pan M, Sheng S, Fan Z, et al. Ultrasound-guided percutaneous release of A1 pulley by using a needle knife: a prospective study of 41 cases. Front Pharmacol. 2019;10:267. doi:10.3389/fphar.2019.00267

10. Park MJ, Oh I, Ha KI. A1 pulley release of locked trigger digit by percutaneous technique. J Hand Surg Br. 2004;29(5):502-505. doi:10.1016/J. JHSB.2004.03.015

11. Guerini H, Pessis E, Theumann N, et al. Sonographic appearance of trigger fingers. J Ultrasound Med. 2008;27(10):1407-1413. doi:10.7863/ jum.2008.27.10.1407

12. Lapègue F, André A, Meyrignac O, et al. US-guided percutaneous release of the trigger finger by using a 21-gauge needle: a prospective study of 60 cases. Radiology. 2016;280(2):493-499. doi:10.1148/radiol.2016151886

13. Boutry N, Titécat M, Demondion X, et al. High-frequency ultrasonographic examination of the finger pulley system. J Ultrasound Med. 2005;24 (10):1333-1339. doi:10.7863/jum.2005.24.10.1333

14. Smith J, Rizzo M, Lai JK. Sonographically guided percutaneous first annular pulley release: cadaveric safety study of needle and knife techniques. J Ultrasound Med. 2010;29(11):1531-1542. doi:10.7863/jum.2010.29.11.1531

15. Paulius KL, Maguina P. Ultrasound-assisted percutaneous trigger finger release: is it safe? Hand (N Y). 2009;4(1):35-37. doi:10.1007/s11552-0089137-8

16. Schramm JM, Nguyen M, Wongworawat MD. The safety of percutaneous trigger finger release. Hand (N Y). 2008;3(1):44-46. doi:10.1007/s11552007-9069-8

17. Wilhelmi BJ, Mowlavi A, Neumeister MW, et al. Safe treatment of trigger finger with longitudinal and transverse landmarks: an anatomic study of the border fingers for percutaneous release. Plast Reconstr Surg. 2003;112(4):993-999. doi:10.1097/01.PRS.0000076225.79854.F7

18. Gil JA, Hresko AM, Weiss AC. Current concepts in the management of trigger finger in adults. J Am Acad Orthop Surg. 2020;28(15):e642-e650. doi:10.5435/JAAOS-D-19-00614

19. Jongjirasiri Y. Length and landmark of A1 pulley in hand: an anatomical study. J Med Assoc Thai. 2009;92(1):41-46.

Journal of Pain Research

Dovepress

\section{Publish your work in this journal}

The Journal of Pain Research is an international, peer reviewed, open access, online journal that welcomes laboratory and clinical findings in the fields of pain research and the prevention and management of pain. Original research, reviews, symposium reports, hypothesis formation and commentaries are all considered for publication. The manuscript management system is completely online and includes a very quick and fair peer-review system, which is all easy to use. Visit http://www.dovepress.com/testimonials.php to read real quotes from published authors.

Submit your manuscript here: https://www.dovepress.com/journal-of-pain-research-journal 